\title{
Regulating synchronous states of complex networks by pinning interaction with an external node
}

\author{
J.A. Almendral, ${ }^{1}$ I. Sendiña-Nadal, ${ }^{1}$ D. Yu, ${ }^{2}$ I. Leyva, ${ }^{1}$ and S. Boccaletti ${ }^{3,4}$ \\ ${ }^{1}$ Complex Systems Group, Universidad Rey Juan Carlos, c/ Tulipan s/n, 28933 Mostoles, Madrid, Spain \\ ${ }^{2}$ College of Automation Engineering, Qingdao University, Qingdao, Shandong 266071, China \\ ${ }^{3}$ Embassy of Italy in Tel Aviv, 25 Hamered St., 68125 Tel Aviv, Israel \\ ${ }^{4}$ CNR-Istituto dei Sistemi Complessi, Via Madonna del Piano, 10, 50019 Sesto Fiorentino (Fi), Italy
}

(Dated: 14/06/2009)

\begin{abstract}
An initial ensemble of networking dynamical systems is regulated towards a desired synchronized behavior by means of a pinning interaction with an external node. We rigorously demonstrate that there are two classes of networks susceptible of being regulated into a synchronous motion, and practically demonstrate, for each one of them, how to design a proper minimal, optimal, and maximal pinning sequence to achieve synchrony regulation. A series of numerical examples are offered to support our analytical claims. We also discuss how the obtained sequences can be compared with a topological ranking of the network nodes, such as centrality, and the practical applications of our study in the understanding of how networking systems can adjust their architecture in order to establish (or maintain) a synchronous functioning.
\end{abstract}

PACS: 05.45.Xt, 89.75.Kd

Regulating the synchronous behavior of networking dynamical units still remains among the fundamental challenges in modern scientific research, especially for biological, physiological and cellular processes, where they are nowadays considered as the key process through which such networks establish and maintain their correct functioning [1]. In physiology, examples include the regulation of the synchronous beating of heart cells by pacemakers in the sinoatrial node [2], or the control of the respiratory rhythm by pacemaker neurons in the medulla [3], or the observed generation of rhythmic currents in the gastrointestinal tract of mammals [4]. In biology, many evidences exist of mechanisms through which cell cycles and behaviors are controlled and synchronized [5].

In this Letter, we give a full analytical treatment of an initial network of identical dynamical units, whose synchronous behavior is regulated by a pinning interaction with an external node, i.e. by the addition of a further identical system forming connections with a limited number of nodes in the original graph. In particular, we will rigorously establish conditions for identifying a minimal, maximal, and optimal number of connections needed for regulating synchrony in a complex network, as well as a practical way to define the corresponding minimal, maximal and optimal sequence of connections. We will furthermore discuss how the regulation process depends on the local dynamics acting on each one of the network's nodes, and how the resulting sequences of connections are related to some of the topological ranking of the nodes in the original graph.

The problem under study can be conveniently described by introducing an initial graph $\mathcal{G}_{0}$ of $N$ bidirectionally coupled identical systems, each one of them represented by a vector state $\mathbf{x}_{i}(t)(i=1, \ldots, N) \in \mathbb{R}^{m}$, whose evolution equation is

$$
\dot{\mathbf{x}}_{i}=f\left(\mathbf{x}_{i}\right)+\sigma \sum_{j=1}^{N} \mathcal{L}_{i j} h\left(\mathbf{x}_{j}\right),
$$

where dot denotes temporal derivative, $\mathcal{L}=\left(\mathcal{L}_{i j}\right) \in \mathcal{M}_{N}$ is the Laplacian matrix associated with the network $\mathcal{G}_{0}$, $f(\mathbf{x}): \mathbb{R}^{m} \rightarrow \mathbb{R}^{m}$ is the function ruling the local evolution, $h(\mathbf{x}): \mathbb{R}^{m} \rightarrow \mathbb{R}^{m}$ is the output function of the node, and $\sigma \in \mathbb{R}^{+}$is the strength of the bidirectional coupling, which, from now on, will be such that the synchronous state $\left[\mathbf{x}_{1}(t)=\mathbf{x}_{2}(t)=\ldots=\mathbf{x}_{N}(t)=\mathbf{x}_{s}(t)\right]$ is linearly unstable.

In order to regulate the stability of $\mathbf{x}_{s}(t)$, we consider here an interaction between $\mathcal{G}_{0}$ and an external dynamical system, identical to those in $\mathcal{G}_{0}$, that forms, at successive times $t_{n}(n=1, \ldots, N)$ a series of $\sigma$-strength connections by pinning the nodes in $\mathcal{G}_{0}$ with a given sequence $\left\{s_{1}, s_{2}, \ldots, s_{N}\right\}$.

The new equation of motion then reads

$$
\dot{\mathbf{x}}_{i}=f\left(\mathbf{x}_{i}\right)+\sigma \sum_{j=1}^{N+1} \mathcal{L}_{i j}^{\prime}(t) h\left(\mathbf{x}_{j}\right),
$$

where $\mathcal{L}^{\prime}(t)=\left(\mathcal{L}^{\prime}{ }_{i j}(t)\right) \in \mathcal{M}_{N+1}$ is now the following time dependent Laplacian matrix 

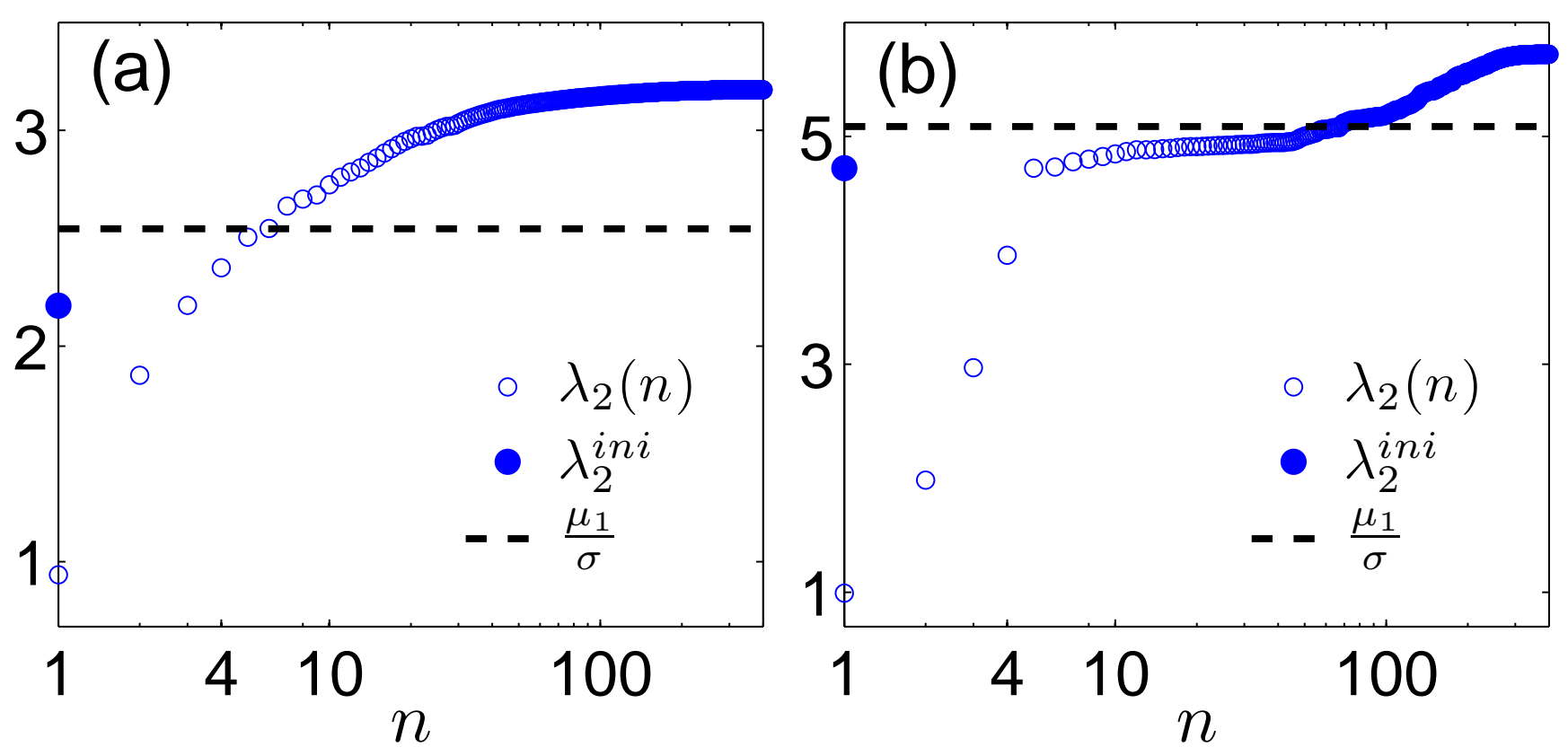

FIG. 1: (Color online) Log-linear graph showing the behavior of $\lambda_{2}(n)$ during the regulation of a Class II system $(f(\mathbf{x} \equiv(x, y, z))$ as reported in the text and $h(\mathbf{x} \equiv(x, y, z))=[0, y, 0]$ for which $\left.\mu_{1}=0.178\right)$ for (a) SW, and (b) SF networks (see text for parameters). As reference, the values of $\lambda_{2}^{i n i}$ and the ratio $\mu_{1} / \sigma$ with (a) $\sigma=0.07$ and (b) $\sigma=0.035$ are given.

$$
\left(\begin{array}{cccc|c}
\mathcal{L}^{\prime}{ }_{11}(t) & \mathcal{L}^{\prime}{ }_{12} & \cdots & \mathcal{L}^{\prime}{ }_{1 N} & \Theta\left(t-T_{1}\right) \\
\mathcal{L}^{\prime}{ }_{21} & \mathcal{L}^{\prime}{ }_{22}(t) & \cdots & \mathcal{L}^{\prime}{ }_{2 N} & \Theta\left(t-T_{2}\right) \\
\vdots & \vdots & \ddots & \vdots & \vdots \\
\mathcal{L}^{\prime}{ }_{N 1} & \mathcal{L}^{\prime}{ }_{N 2} & \cdots & \mathcal{L}^{\prime}{ }_{N N}(t) & \Theta\left(t-T_{N}\right) \\
\hline \Theta\left(t-T_{1}\right) & \cdots & \cdots & \Theta\left(t-T_{N}\right) & \mathcal{L}^{\prime}{ }_{N+1}, N+1 \\
& & & &
\end{array}\right)
$$

whose elements are such that:

i) $\mathcal{L}^{\prime}{ }_{i j}=\mathcal{L}_{i j}$ for $i \neq j$ and $i, j<N+1$;

ii) $\Theta\left(t-T_{i}\right)=\mathcal{L}^{\prime}{ }_{i, N+1}(t)=\mathcal{L}^{\prime}{ }_{N+1, i}(t)$, being $T_{i}$ the time at which the $i^{t h}$ node in $\mathcal{G}_{0}$ is pinned by the interaction with the external node, and $\Theta$ the Heaviside function. Notice that while the index $i$ in $t_{i}$ refers to a time ordering, the index $i$ in $T_{i}$ points to the ordering of the pinning sequence, and therefore $t_{i}=T_{s_{i}}$.

iii) $\mathcal{L}^{\prime}{ }_{i i}(t)=-\sum_{j \neq i} \mathcal{L}^{\prime}{ }_{i j}$.

The key point is the way the sequence $\left\{s_{1}, s_{2}, \ldots, s_{N}\right\}$ is selected to ensure regulation of the synchronous behavior of $\mathcal{G}_{0}$. Since the coupling matrix $\mathcal{L}^{\prime}(t)$ is a symmetric, zero-row sum matrix at all times, one can make use of the arguments of the Master Stability Function (MSF) approach [6] to assess the stability property of the new invariant synchronous manifold $\mathbf{x}_{1}(t)=\mathbf{x}_{2}(t)=\ldots=\mathbf{x}_{N+1}(t)=\mathbf{x}_{s}(t)$ of Eq. (1).

In particular, Ref. [7] fully discusses the application of the MSF formalism to a generic complex network's architecture, and demonstrates that, depending on the specific choice of the functions $f(\mathbf{x})$ and $h(\mathbf{x})$, only two possible classes of dynamical systems allow stability of a synchronous network's behavior: the so-called class II systems, where the stability of the synchronous state is guaranteed above a given threshold in the coupling strength $\sigma$, and class III systems, where the stability of the synchronous state is obtained within a given interval of coupling strength values [8]. In the former case, the role of the network's architecture is that of setting a threshold $\sigma_{c} \equiv \frac{\mu_{1}}{\lambda_{2}}$, with $\mu_{1}$ being the value of the MSF parameter at which the MSF itself passes from positive to negative values of the conditional maximum Lyapunov exponent [7], and $\lambda_{2}$ is the second smallest eigenvalue of the network's coupling matrix. In the latter case, class III systems, the synchronous network behavior is stable between $\mu_{1}$ and $\mu_{2}>\mu_{1}$, so that the network 

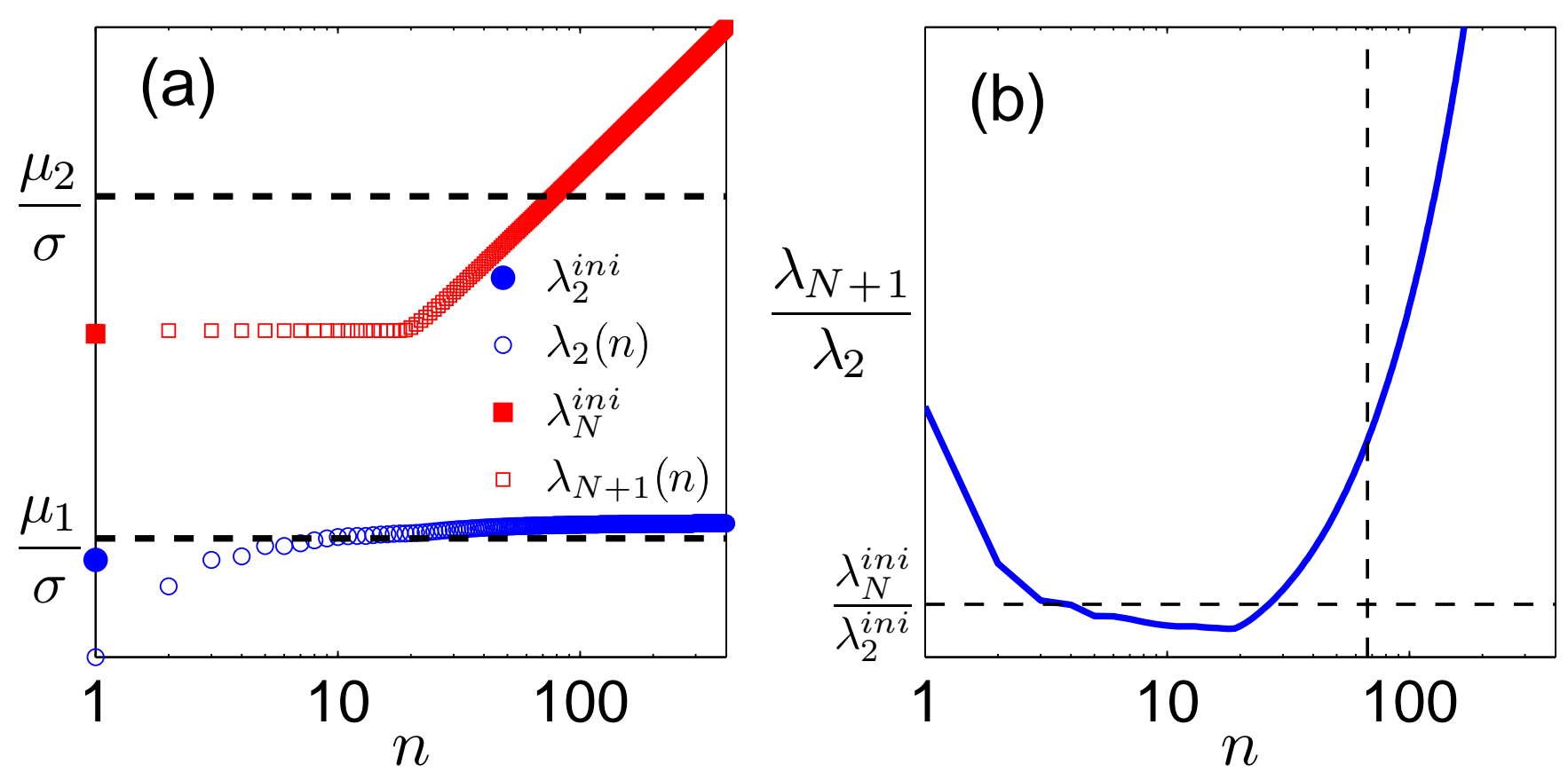

FIG. 2: (Color online) (a) Log-log behavior of $\lambda_{2}(n)$ (blue open circles) and $\lambda_{N+1}(n)$ (red open squares) for the regulating process of a SW network (see text for parameters). Full symbols denote the values $\lambda_{2}^{i n i}$ and $\lambda_{N}^{i n i}$. Dashed lines are in correspondence with the two thresholds $\mu_{1} / \sigma$ and $\mu_{2} / \sigma$ (see text for parameters). (b) Linear-log behavior of the ratio $\frac{\lambda_{N+1}(n)}{\lambda_{2}(n)}$. The horizontal dashed line denotes the ratio $\lambda_{N}^{i n i} / \lambda_{2}^{i n i}$ of $\mathcal{G}_{0}$ and the vertical dashed line is in correspondence with the minimal number $n_{m i n}$ of links for which $\lambda_{N+1}\left(n_{\text {min }}\right)$ in panel (a) crosses the threshold $\mu_{2} / \sigma$.

capability to sustain a synchronized dynamics is fully accounted for by the ratio between the largest and the second smallest eigenvalue in the spectrum of the network's coupling matrix, $\frac{\lambda_{N+1}}{\lambda_{2}}$.

These two different classes of functions need here to be treated separately. Indeed, in previous approaches of pinning control of networks [9] (i.e. the situation in which the external node is unidirectionally forcing the dynamics of the original graph), it was argued that the controllability of a generic network behavior towards an assigned synchronous evolution could be enhanced by pinning configurations that imply a decreasing of the ratio $\frac{\lambda_{N+1}}{\lambda_{2}}$ associated to the extended network topology [10]. However, we will see momentarily that the concept of regulation of synchrony described here is fully different from that of network controllability, and we will show that, for class III systems, many pinning sequences exist that are able to regulate the synchronous behavior of $\mathcal{G}_{0}$ and that, however, correspond to values of $\frac{\lambda_{N+1}}{\lambda_{2}}$ that are larger than the initial (unpinned) value, for which the synchronous behavior was unstable.

From here on, we will accompany our analytical treatment with numerical examples, corresponding to the case of $N=400$ nodes arranged in two different network configurations: a small-world network (SW) obtained as in Ref. [11] by initially arranging the $N$ nodes in a ring with connections only between nearest neighbors, and by randomly adding with probability $p=0.02$ a connection between unconnected pairs of nodes (i.e. obtaining an average degree $\langle k\rangle=2+p N=10$ ), and a scale-free network (SF) obtained by the preferential attachment process of Ref. [12] with the same average degree of $\langle k\rangle=10$. Furthermore, in all cases, we will consider $f(\mathbf{x} \equiv(x, y, z))=[-y-z, x+$ $0.165 y, 0.2+z(x-10)]$ in Eq. (1) (i.e. we will refer to the case of networks of coupled Rössler systems [13]), because it is known that such a case allows for a direct comparison of class III networks (when $h(\mathbf{x} \equiv(x, y, z))=[x, 0,0])$ and class II networks (when $h(\mathbf{x} \equiv(x, y, z))=[0, y, 0])$.

With these stipulations, let us proceed first with the analysis of the class II system. The initial configuration is an unstable synchronous motion in which $\lambda_{2}^{\text {ini }}<\frac{\mu_{1}}{\sigma}$ being $\lambda_{2}^{\text {ini }}$ the second smallest eigenvalue of $\mathcal{L}$ [i.e. $\lambda_{3}$ of $\left.\mathcal{L}^{\prime}(0)\right]$. Since the larger is $\lambda_{2}$, the smaller the threshold for setting a stable synchrony, a practical way to regulate the synchronous state is as follows: at time $t_{1}$ (at which the first connection is established between the regulating node and $\mathcal{G}_{0}$ ) we consider all possible choices and fix $s_{1}$ as the index of the node for which one obtains the maximum value for the second smallest eigenvalue $\lambda_{2}(1)$ in the matrix $\mathcal{L}^{\prime}\left(T_{s_{1}}\right)$. After establishing this connection, at time $t_{2}$ one looks for that index $s_{2} \neq s_{1}$ for which again the matrix $\mathcal{L}^{\prime}\left(T_{s_{2}}\right)$ displays a maximum value of $\lambda_{2}(2)$. At time $t_{3}$ the process is repeated to select $s_{3} \neq s_{1}, s_{2}$, and so on, up to the point at which the full sequence $\left\{s_{1}, s_{2}, \ldots, s_{N}\right\}$ is determined. 

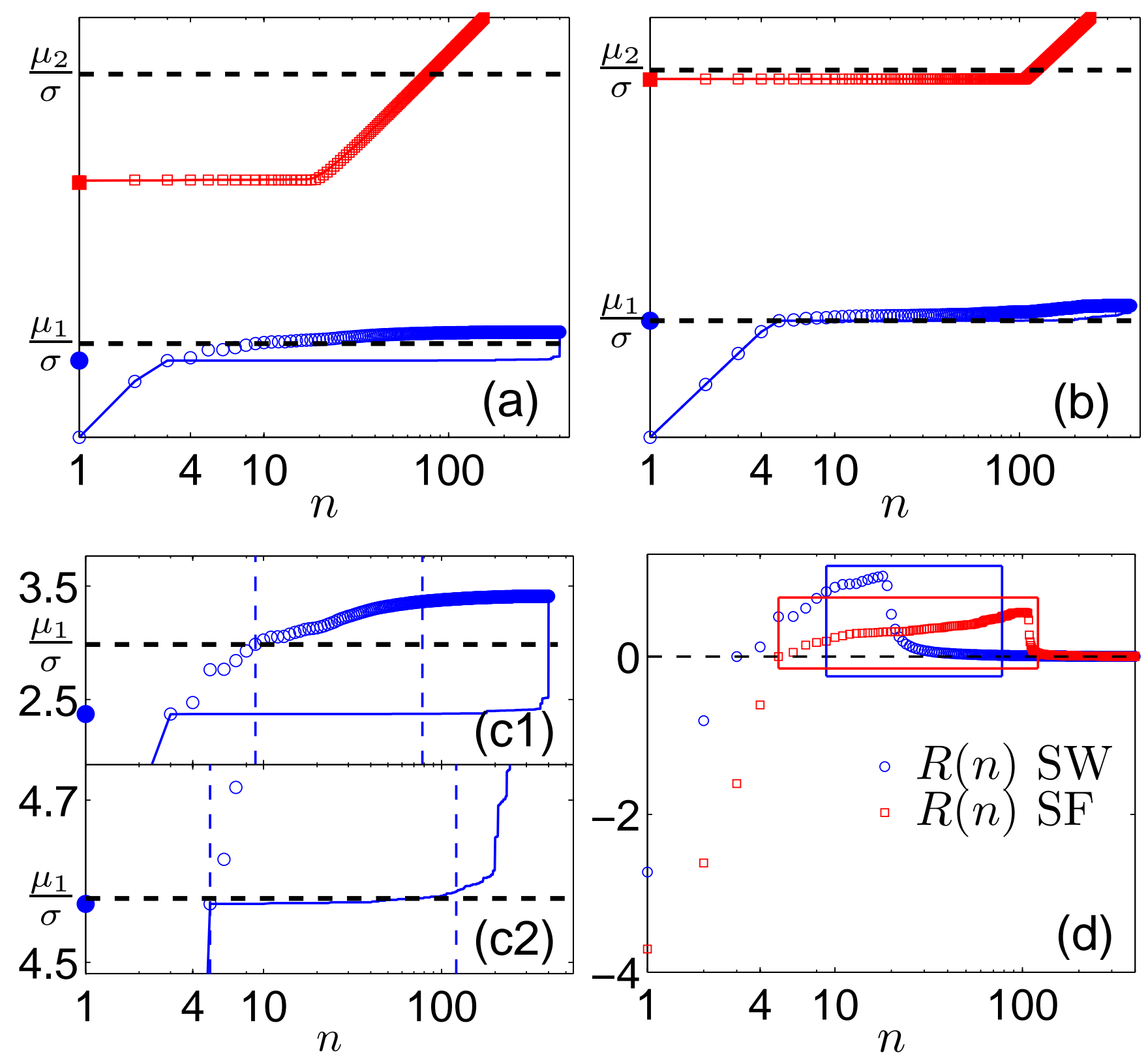

FIG. 3: (Color online) (a)-(b) Log-log behavior of $\lambda_{2}(n)$ (blue open circles) and $\lambda_{N+1}(n)$ (red open squares) during regulation of a class III (a) SW and (b) SF network (same parameters and stipulations as in Fig. 1). For comparison, the two panels report also $\lambda_{2}^{c}(n)\left[\lambda_{N+1}^{c}(n)\right]$ as a blue (red) solid line, as obtained from a ranking centrality-based pinning regulation process. The values of $\lambda_{2}^{i n i}$ (blue full circle) and $\lambda_{N}^{i n i}$ (red full square) refer to those of $\mathcal{G}_{0}$, and the horizontal dashed lines are in correspondence with the limiting thresholds $\mu_{1} / \sigma$ and $\mu_{2} / \sigma$ with (a) $\sigma=0.07$ and (b) $\sigma=0.035$. Panel (c) shows the zooms of the $\lambda_{2}(n)$ behavior for a SW (c1), and for a SF (c2) networks, with vertical dashed lines marking the initial and final values of $n$ for which the networks are regulated. (d) Log-linear behavior of $R(n)$ (see text for definition) for the previous SW (blue circles) and SF (red squares) networks. The red and blue rectangles denote the range of $n$ in which the networks are synchronized due to regulation.

Figure 1 reports the values of the second smallest eigenvalue $\lambda_{2}(n)$ vs. the number $n$ of links formed by the regulating node with nodes in $\mathcal{G}_{0}$, for the above described procedure applied to the SW [panel (a)] and SF [panel (b)] networks, and shows that the method allows, indeed, for the identification, in both cases, of a minimal number (and corresponding minimal sequence) of links needed for the regulation of the synchronous state. A synoptic view of the two panels in Fig. 1 reveals that, in class II, SW networks are easier to regulate with respect to SF ones in that the corresponding minimal number of links to make the synchronous state stable is smaller, and that synchronizability is 

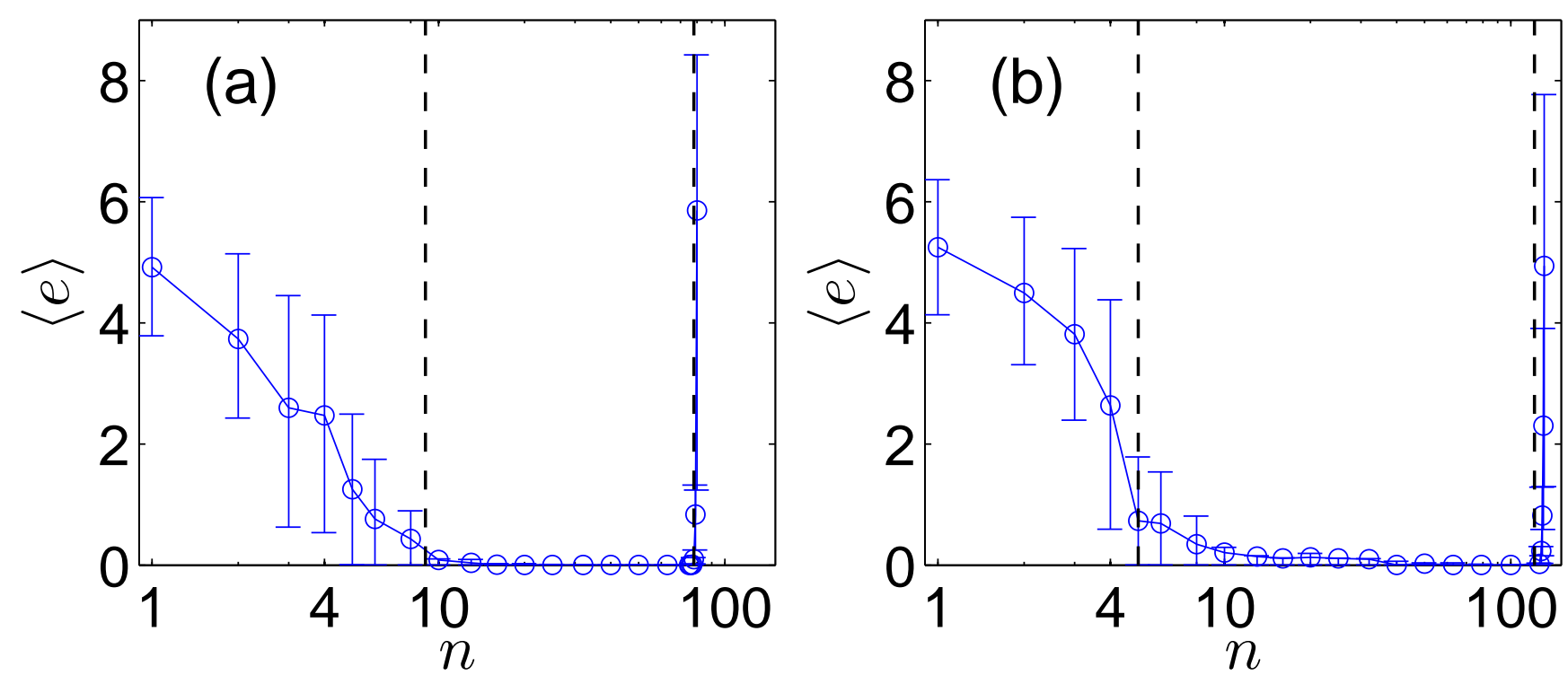

FIG. 4: Log-linear plots of the averaged synchronization error $\langle e\rangle$ (see text for definition) vs. $n$ during the regulation of the same (a) SW and (b) SF networks used in Fig. 3 and the same pinning sequence optimizing $R(n)$ [Fig. 3(d)]. Vertical dashed lines are added to show the agreement with the predicted range of regulability depicted in Fig. 3(d).

monotonically enhanced with $n$ as the behavior of the synchronization threshold $\frac{\mu_{1}}{\lambda_{2}(n)}$ is a decreasing function of $n$. These results, therefore, are fully consistent with what recently reported in a degree driven pinning control process of class II networks [14], for which the existence of a threshold for the number of nodes needed for control was observed.

The situation is completely different in the case of class III networks. Let us start from an unstable synchronous motion in which $\lambda_{2}^{i n i}<\frac{\mu_{1}}{\sigma}$ and $\lambda_{N}^{i n i}<\frac{\mu_{2}}{\sigma}$, being $\lambda_{N}^{i n i}$ the largest eigenvalue of $\mathcal{L}$ [i.e. $\lambda_{N+1}$ of $\mathcal{L}^{\prime}(0)$ ]. Here, the synchronization stability is ensured for all coupling architectures whose corresponding eigenvalue spectrum is entirely contained within the stability region of the MSF, delimited by the two threshold parameters $\mu_{1}$ and $\mu_{2}$. Then, the way we select the pinning sequence is that to maximize (at each time $t_{n}$ a new link is formed with the regulating node) the quantity

$$
R(n)=\frac{\lambda_{2}(n)-\lambda_{2}^{i n i}}{\lambda_{N+1}(n)-\lambda_{N}^{i n i}}
$$

In practice, at time $t_{1}$, out of all possible choices, $s_{1}$ is the index of the node maximizing $R(1)$. After having established this connection, at time $t_{2}$ one looks for that $s_{2} \neq s_{1}$ for which the spectrum of $\mathcal{L}^{\prime}\left(T_{s_{2}}\right)$ displays a maximum of $R(2)$, and so on, up to when the regulating sequence $\left\{s_{1}, s_{2}, \ldots, s_{N}\right\}$ is determined [15].

It is important to remark that this regulating procedure is a completely different approach with respect to that of pinning control [10], as it is evident from inspection of Fig. 2. There, the regulating procedure is applied to the same SW network of Fig. $1(\mathrm{a})$ but with $h(\mathrm{x} \equiv(x, y, z))=[x, 0,0]$, which determines $\mu_{1}=0.206$ and $\mu_{2}=5.519$ in the considered system. Panel (a) of the figure shows that an initial unsynchronized motion in $\mathcal{G}_{0}$ (see the blue full circle and red full square) is effectively regulated for all sequences from $n \sim 10$ (when the blue circles enter the stability region) to $n \sim 70$ (where the red squares exit from the stability region), and synchrony looses again stability. However, the behavior of the eigenratio $\frac{\lambda_{N+1}(n)}{\lambda_{2}(n)}$ [reported in Fig. 2(b)] is not monotonic, and it is evident that a huge number of regulating sequences (those from $n \sim 25$ to $n \sim 70$ ) correspond to eigenratio values that are even larger than $\lambda_{N}^{i n i} / \lambda_{2}^{i n i}$ (at which $\mathcal{G}_{0}$ displayed an unsynchronous motion).

A full account of the regulating process for class III networks is presented in Fig. 3. Namely, panels (a) and (b) report the behavior of $\lambda_{2}(n)$ and $\lambda_{N+1}(n)$ at all stages of the selection of the regulating sequence for the mentioned SW and the SF networks. In both cases, and at variance with the class II case, class III networks are characterized by the possibility for regulation only from a minimum to a maximum length of the regulating sequence, and a comparative inspection of the vertical blue dashed lines of panels (c1) and (c2) (delimiting the ranges in which regulation is effective), shows that now SF architectures allow for a larger range of sequences, the limits to the sequence length being here determined by the monotonic growth of $\lambda_{N+1}(n)$, which eventually determines the emergence of the second instability for synchronization. 
The blue and red solid lines in Figs. 3(a-c) allow for a direct comparison of the regulating capability of the sequences selected by our procedure with $\lambda_{2}^{c}(n)$ and $\lambda_{N+1}^{c}(n)$, corresponding to the sequences obtained by pinning the nodes of $\mathcal{G}_{0}$ following a ranking based on the eigenvector centrality [16]. In all cases, our sequencing leads to a more effective regulating mechanism, indicating that our approach can be used as a novel way for ranking nodes in a network depending on their importance for regulating the graph's collective functioning. Thus, this method, allows for gathering information on network's nodes that is not contained in other topologically based ranking methods. Furthermore, Fig. 3(d) reports the behavior of $R(n)$ for the two networks (with red and blue rectangles delimiting the range of regulability), and shows how in both cases one can define an optimal sequence for regulation in correspondence with the appearance of the maximum in the curve of $R(n)$ (which corresponds to the regulating subsequence that better positions the entire spectrum of the network coupling matrix within the synchronizability range).

Finally, in order to verify the set of analytical findings presented here, we monitor during the regulating process the vanishing of the time average synchronization error $e=\frac{1}{T N} \sum_{j=1, \ldots, N} \int_{t}^{t+T}\left\|\mathbf{x}_{j}-\mathbf{x}_{N+1}\right\|_{2} \mathrm{~d} t$ over a window $T=120$ t.u. In Fig. 4 we plot the averaged error $\langle e\rangle$ for 200 different initial conditions of the networking systems (together with their standard deviation as error bars), to show how accurately the predicted regulating ranges are numerically verified.

In conclusion, we have rigorously demonstrated that an initial network of identical uncorrelated dynamical systems can be regulated towards its synchronized motion by means of a pinning interaction of the network with a further external node, and we have given the conditions for devising proper minimal, optimal, and maximal pinning sequences to achieve synchrony regulation. Besides giving practical ways of devising regulating architecture, the proposed technique allows for a dynamical-based ranking of nodes in a graph, following their role in a regulating process of the graph's collective functioning, that can shed, therefore, new light on the way biological systems grow and rearrange their connectivity architecture in order to establish and/or maintain a synchronous functioning.

Work partly supported by EU contract 043309 GABA, by the URJC-CM under Projects Nos. 2007-CET-1601 and 2008-CET-3575, and by the Chinese NSFC under Grant No. 10602026.

[1] S.V. Vishnampet, Regulation and Control Mechanisms in Biological Systems, (Prentice Hall, 1992).

[2] C. Peskin, Mathematical aspects of heart physiology (Courant Inst. Math Sci., New York, 1977).

[3] N. Koshiya and J.C. Smith, Nature 400, 360 (1999).

[4] J. Thomsen et al., Nature Medicine 4, 848 (1998).

[5] R. Hunt, A. Hunter, and A. Munro, Nature 220, 481 (1968); L.H. Hartwell, J. Culotti, and B. Reid, P.N.A.S. 66, 352 (1970); P. Nurse, Nature 256, 547 (1975); D. Battogtokh, K. Aihara, and J. Tyson, Phys. Rev. Lett. 96, 148102 (2006).

[6] L. Pecora and T. Carroll, Phys. Rev. Lett. 80, 2109 (1998).

[7] S. Boccaletti, V. Latora, Y. Moreno, M. Chavez, and D. Hwang, Physics Reports 424, 175 (2006).

[8] We do not consider class I systems, as they are unable to network synchronously for any graph architecture and, in this sense, they are not susceptible of being regulated.

[9] R. Grigoriev, M. Cross, and H. Schuster, Phys. Rev. Lett. 79, 2795 (1997); N. Parekh, S. Parthasarathy, and S. Sinha, Phys. Rev. Lett. 81, 1401 (1998); X. Wang and G. Chen, Physica A 310, 521 (2002).

[10] F. Sorrentino, M. di Bernardo, F. Garofalo, and G. Chen, Phys. Rev E 75, 046103 (2007).

[11] M.E.J. Newman and D.J. Watts, Phys. Rev. E 60 (1999) 7332.

[12] A.-L. Barabási and R. Albert, Science 286, 509 (1999).

[13] O.E. Rössler, Phys. Lett. 57A, 397 (1976).

[14] Y. Zou and G. Chen, Europhys. Lett. 84, 58005 (2008).

[15] Any procedure consisting in adding links to $\mathcal{G}_{0}$ will never decrease the largest eigenvalue of the Laplacian matrix, and therefore, if the initial configuration of $\mathcal{G}_{0}$ is such that $\lambda_{N}^{i n i}>\frac{\mu_{2}}{\sigma}$ the pinning regulation becomes impossible.

[16] M.E.J. Newman, SIAM Rev. 45, 167 (2003). 